\title{
Estimation of time since death using a body cooling model of pigs: A pilot study
}

\begin{tabular}{l}
\hline Paper received: 15.09 .2018 \\
\hline \\
Authors Info \\
C.H. Hyun', T.H. Song ${ }^{2}$ K.Y. Sim ${ }^{3}$, \\
N.J. Kim, D.S. Kim ${ }^{5}$ and \\
D.K. Park ${ }^{5 *}$ \\
${ }^{1}$ Jeonbuk Provincial Police Agency, \\
Jeonju, 54962, Republic of Korea \\
${ }^{2}$ Korea Police Investigation \\
Academy, Asan, 31540, Republic \\
of Korea \\
${ }^{3}$ Gyeonggi Nambu Provincial Police \\
Agency, Suweon, 16218, Republic \\
of Korea \\
${ }^{4}$ Busan Metropolitan Police Agency, \\
Busan, 47545, Republic of Korea \\
${ }^{5}$ Department of Anatomy, \\
Soonchunhyang University, \\
Cheonan, 31151, Republic of \\
Korea \\
\end{tabular}

\section{Edited by \\ Dr. Sumati Gaumat}

\section{Reviewed by}

Professor Seong-Ho Ryu

Professor Yi-Suk Kim

\section{Abstract}

Aim : The aim of this study was to test the effects of Korean floor-heating system on heat loss using adult pig models, and to create a novel formula for estimating time since death during the early stages of decomposition.

Methodology : Three electric mattress pads were placed on the ground to maintain a constant temperature of the substrate like the ondol heating system. Four temperature measuring probes were placed in each pig: inside the rectum, on the body surface, between the body and the surface of mattress pad and on the mattress pad. The probes were connected to a temperature data logger system. Temperature was recorded every minute and statistical analysis was performed using the SAS (version 9.3) program.

Results : Spearman's Rank Correlation results demonstrated the rectal temperature, and the temperature between the body and the surface of pad were strongly correlated with postmortem cooling of the body, rather than ambient temperature. The rate of cooling of the body is represented by a cube function of time rather than an exponential or bi-exponential function.

Interpretation: This research indicates that postmortem cooling of the body is more influenced by ground surface temperature than by ambient (environment) temperature, and the rectal temperature fluctuated with the ambient temperature. Additionally, the study showed that pigs can be good animal models that can substitute human cadaver to study the process of decomposition.

Key words: Animal model, Body cooling, Pig, Postmortem interval

How to cite : Hyun, C.H., T.H. Song, K.Y. Sim, N.J. Kim, D.S. Kim and D.K. Park: Estimation of time since death using a body cooling model of pigs: A pilot study. J. Environ. Biol., 40, 265-270 (2019). 


\section{Introduction}

In forensic studies it is very important to determine the time since death at early stages of decomposition in daily casework. Algor mortis is the term used for the change in body temperature at early stage of decomposition, until the body temperature has reached the ambient (environment) temperature. Earlier, the most accurate way to measure body temperature was rectal temperature. Henssge reported that rectal temperature was cooled as dependence of the ambient temperature using two exponential equations of Marshall and Hoare (Henssge, 1988; Marshall and Hoare, 1962). Algor mortis can be used to determine the time since death, based on this study.

Henssge nomogram was established by the correlation between postmortem cooling of the body, rectal temperature, ground temperature, and ambient temperature, which is essential for estimating time since death at early stages of decomposition (Henssge, 1988; Henssge, 1992; Althaus and Henssge, 1999; Henssge et al., 2000 a, b; Henssge and Madea, 2004; Henssge and Madea, 2007). The Korean Police Investigation Academy (KPIA) recommends that crime scene investigators employ the Henssge nomogram method at death scene, which is estimated at time since death using rectal and ambient temperature. However, the application of Henssge nomogram is problematic because bodies are commonly found indoors in Korea. Moreover, there is a paucity of research on the effects of heat radiation and Henssge nomogram. In view of the above, the present study was conducted to test the effects of Korean floor-heating system on heat loss using adult pig models, and to create a novel formula for estimating time since death during early stages of decomposition.

\section{Materials and Methods}

Four $40 \mathrm{~kg}$ pigs (Sus scrofa) were killed by administering euthanasia drug for animals following Soonchunhyang University Institutional Animal Care and Use Committee protocol. Three electric mattress pads were placed on the ground to maintain a constant temperature of the substrate like the ondol heating system. Each pig was placed on four different surfaces; low-heat, medium-heat and high-heat electric mattress pads and ground (that served as control). Four temperature measuring probes were placed in each pig (Fig 1): inside the rectum, on the body surface, between the body and the surface of mattress pad or ground (control), and on the mattress pad or ground (control). The probes were connected to a temperature data logger system and Watchdog 2000 ministation was located at the research site to measure the ambient temperature. Temperature was recorded every minute and pigs were monitored hourly for $72 \mathrm{hrs}$ by CCTV. Statistical analysis was performed using SAS (version 9.3) program.

\section{Results and Discussion}

The data of four temperature measuring probes are summarized in Fig 2. If the rectal temperature was only influenced by ambient temperature, then the rectal temperature cooling curve should be similar regardless of where the bodies were laid. However, the rectal temperature cooling curve were not same. The rectal temperature of control which was laid on the ground cooled faster than that of experimental group which was laid on the electric mattress. Once the rectal temperature reached that of the ambient temperature, the rectal temperature fluctuated thereafter. As the ambient temperature rose, the rectal temperature rose 2 to 3 hours later, and when the ambient temperature fell, the rectal temperature fell 2 to 4 hours later (Fig 3 ).

The results of Spearman's Rank Correlation are listed in Table 1. The correlation values between the rectal temperature (T1) and with the temperature between the body and the surface of pad or ground (T2) were 0.998 (control), 0.999 (low-heat), 0.981 (mid-heat), 0.979 (high-heat) $(p<0.0001)$. However, the correlation values between the rectal temperature (T1) and the

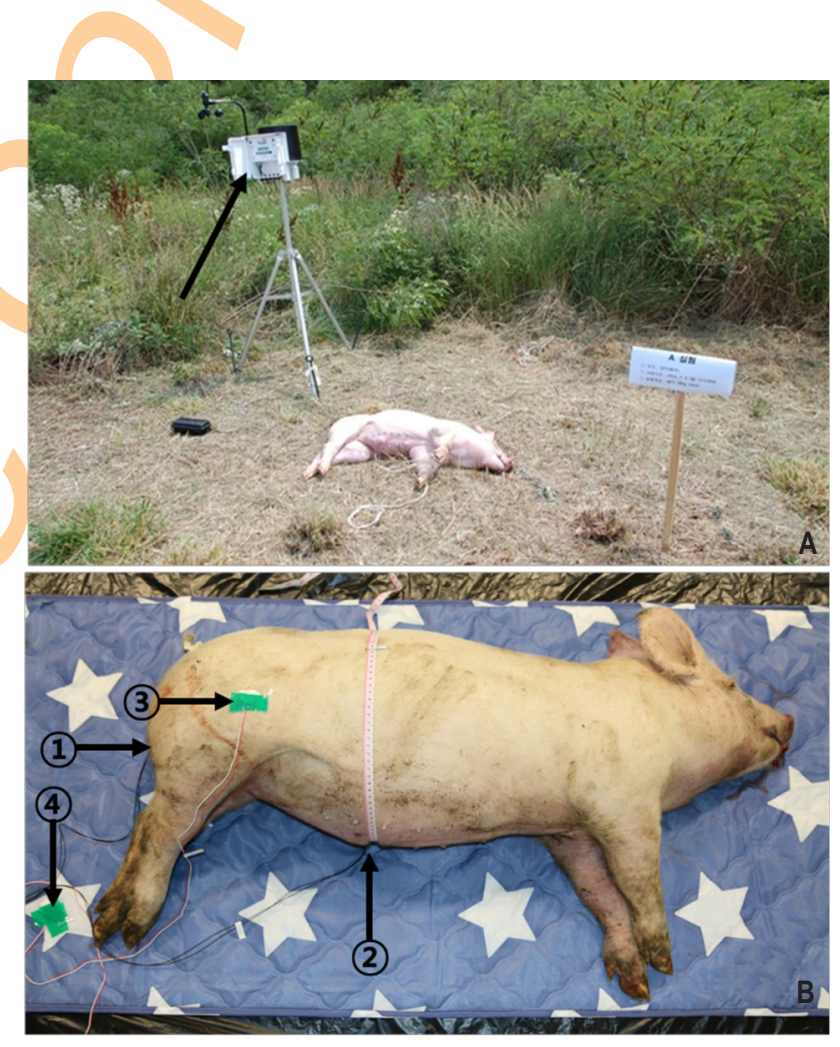

Fig. 1: Photographs of the field set-up for estimating time since death using pigs models. (A) Photograph showing an example of control group. A pig was laid down on the ground. In the upper left quadrant, Watchdog 2000 mini station (arrow) was located at the research site to measure the ambient temperature. (B) Photograph showing an example of experimental group. A pig was laid down on the electric mattress. Three pigswere placed on three different conditions; low-heat, medium-heat and high-heat. Four temperature measuring probes were placed in each pig and connected to a temperature data logger system. (1)-inside the rectum, (2)-between the body and the surface of mattress pad, (3)-on the body surface, and (4)-on the mattress pad. 
ambient temperature (T5) were -0.078 (control), -0.080 (lowheat), -0.084 (mid-heat), -0.086 (high-heat). Therefore, rectal temperature was strongly correlated with the temperature between the body and the surface of pad or ground, rather than the ambient temperature.

The rate of cooling of the body is represented by a cubic function of time rather than an exponential or bi-exponentia function (Fig 4; Table 2.). The results of this pilot study were summarized into three aspects comparing the previous studies (Henssge, 1988; Henssge, 1992; Althaus and Henssge, 1999; Henssge et al., 2000 a, b; Henssge and Madea, 2004; Henssge and Madea, 2007). The main result of this study is that postmortem cooling of rectal temperature is strongly correlated with the temperature between the body and the surface where the body was laid. It indicates that postmortem cooling of the body is more influenced by ground surface temperature (radiation heat from the surface) than by ambient temperature.

Ondol is a traditional floor-heating system in Korea, where the floor is heated from below and the heat radiates to warm the room. Bodies are commonly found indoors in Korea, and the application of Henssge nomogram is problematic due to the effects of ondol heating system on the rate of postmortem heat loss in the body. Investigator need to employ another method instead of Henssge nomogram method at death scene during the early stages of decomposition (Megyesi et al., 2005). Meanwhile, rectal temperature measurement maneuver requires a meticulous placement of thermometer, so it is very hard to measure correctly. However, it is relatively easy to measure the temperature between the body and the surface for non-skilled personnel. Secondly, once the rectal temperature reached the ambient temperature, the rectal temperature can be fluctuated by the ambient temperature. Earlier, studies were (Henssge, 1988;
Table 1 : Results of the Spearman coefficient of correlation using the SAS (version 9.3) program

\begin{tabular}{llllll}
\hline $\begin{array}{l}\text { Coefficient of } \\
\text { Correlation } \\
\text { (SPEARMAN) }\end{array}$ & & Control & $\begin{array}{l}\text { Temp } \\
\mathbf{2 0}{ }^{\circ} \mathrm{C}\end{array}$ & $\begin{array}{l}\text { Temp } \\
\mathbf{2} 5^{\circ} \mathrm{C}\end{array}$ & $\begin{array}{l}\text { Temp } \\
\mathbf{3 0} 0^{\circ} \mathrm{C}\end{array}$ \\
\hline Time & T1 & -1.000 & -1.000 & -0.999 & -0.997 \\
& T2 & -1.000 & -1.000 & -0.999 & -0.993 \\
& T3 & -0.870 & -0.879 & -0.882 & -0.856 \\
& T4 & 0.072 & 0.345 & 0.189 & 0.258 \\
T1 & T5 & 0.078 & 0.080 & 0.082 & 0.084 \\
& T2 & 1.000 & 1.000 & 0.998 & 0.991 \\
& T3 & 0.870 & 0.878 & 0.880 & 0.852 \\
& T4 & -0.072 & -0.345 & -0.189 & -0.261 \\
& T5 & -0.078 & -0.080 & -0.084 & -0.086 \\
T2 & T1 & 1.000 & 1.000 & 0.998 & 0.991 \\
& T3 & 0.870 & 0.878 & 0.880 & 0.848 \\
& T4 & -0.072 & -0.345 & -0.190 & -0.273 \\
& T5 & -0.078 & -0.080 & -0.085 & -0.098 \\
& T1 & 0.870 & 0.878 & 0.880 & 0.852 \\
T3 & T2 & 0.870 & 0.878 & 0.880 & 0.848 \\
& T4 & 0.318 & NS & 0.093 & 0.083 \\
& T5 & 0.285 & 0.262 & 0.240 & 0.296 \\
& T1 & -0.072 & -0.345 & -0.189 & -0.261 \\
& T2 & -0.072 & -0.345 & -0.190 & -0.273 \\
T4 & T3 & 0.318 & NS & 0.093 & 0.083 \\
& T5 & 0.963 & 0.922 & 0.919 & 0.924 \\
\hline
\end{tabular}

T1: temperature inside the rectum, T2: temperature between the body and the surface of pad or ground, T3: temperature of the body surface, T4: temperature of the surface of ground, T5: ambient temperature

Henssge, 1992; Althaus and Henssge, 1999; Henssge et al., 2000 a, b; Henssge and Madea, 2004; Henssge and Madea, 2007) carried out with dummy. So once the temperature of dummy

Table 2: Results of the statistical analysis using the SAS (version 9.3) program

\begin{tabular}{|c|c|c|c|c|c|c|c|}
\hline & & \multicolumn{2}{|c|}{$\mathrm{T} 1{ }^{*} \mathrm{~T} 2$} & \multicolumn{2}{|c|}{$\mathrm{T} 1 * \mathrm{~T} 5$} & \multicolumn{2}{|c|}{$\mathrm{T} 1{ }^{*} \mathrm{~T} 2{ }^{*} \mathrm{~T} 5$} \\
\hline & & $\begin{array}{l}\text { Adjusted } \\
\text { R-Square }\end{array}$ & RMSE & $\begin{array}{l}\text { Adjusted } \\
\text { R-Square }\end{array}$ & RMSE & $\begin{array}{l}\text { Adjusted } \\
\text { R-Square }\end{array}$ & RMSE \\
\hline & Linear Model & 0.888 & 216.763 & 0.783 & 301.427 & 0.894 & 211.127 \\
\hline \multirow[t]{3}{*}{ Control } & Quadratic Model & 0.942 & 156.400 & 0.942 & 155.434 & 0.996 & 42.089 \\
\hline & Cubic Model & 0.997 & 37.070 & 0.986 & 75.805 & 0.999 & 14.849 \\
\hline & Linear Model & 0.913 & 190.754 & 0.799 & 209.005 & 0.966 & 120.033 \\
\hline \multirow[t]{3}{*}{ Temp $20^{\circ} \mathrm{C}$} & Quadratic Model & 0.973 & 106.657 & 0.955 & 137.020 & 0.995 & 44.561 \\
\hline & Cubic Model & 0.996 & 41.139 & 0.991 & 961.226 & 0.998 & 25.932 \\
\hline & Linear Model & 0.933 & 167.732 & 0.780 & 303.891 & 0.961 & 127.142 \\
\hline \multirow[t]{3}{*}{ Temp $25^{\circ} \mathrm{C}$} & Quadratic Model & 0.978 & 95.892 & 0.926 & 175.709 & 0.992 & 57.649 \\
\hline & Cubic Model & 0.996 & 42.867 & 0.942 & 155.54 & 0.996 & 40.471 \\
\hline & Linear Model & 0.894 & 210.684 & 0.743 & 327.861 & 0.923 & 180.113 \\
\hline \multirow[t]{2}{*}{ Temp $30^{\circ} \mathrm{C}$} & Quadratic Model & 0.953 & 139.667 & 0.898 & 206.756 & 0.984 & 83.075 \\
\hline & Cubic Model & 0.986 & 77.385 & 0.962 & 126.495 & 0.996 & 39.606 \\
\hline
\end{tabular}

Adjusted R-square showed large value in cubic model, and RMSE (root mean square error) showed small value in cubic model. The rate of cooling of the body is represented by a cubic function of time rather than an exponential or bi-exponential function. T1: temperature inside the rectum, T2: temperature between the body and the surface of pad or ground, T5: ambient temperature 

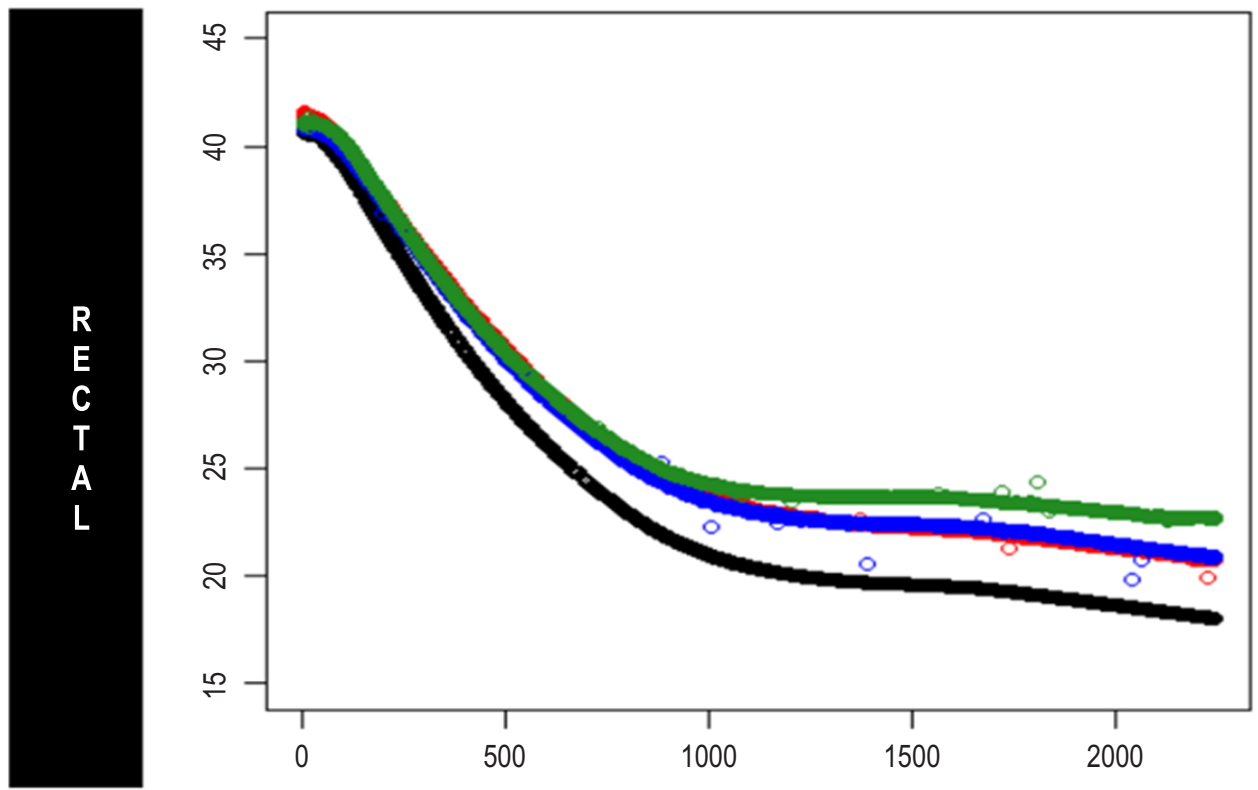

Fig. 2: Results of cooling curve analysis using rectal temperature. Four cooling curves were identified. If the rectal temperature was only influenced by ambient temperature, then one cooling curve were identified. However, the rectal temperature of control group which was laid on the ground cooled faster than that of experimental group which was laid on the electric mattress. Black-control; Red-low-heat;Blue- medium-heat; and Green- high-heat.

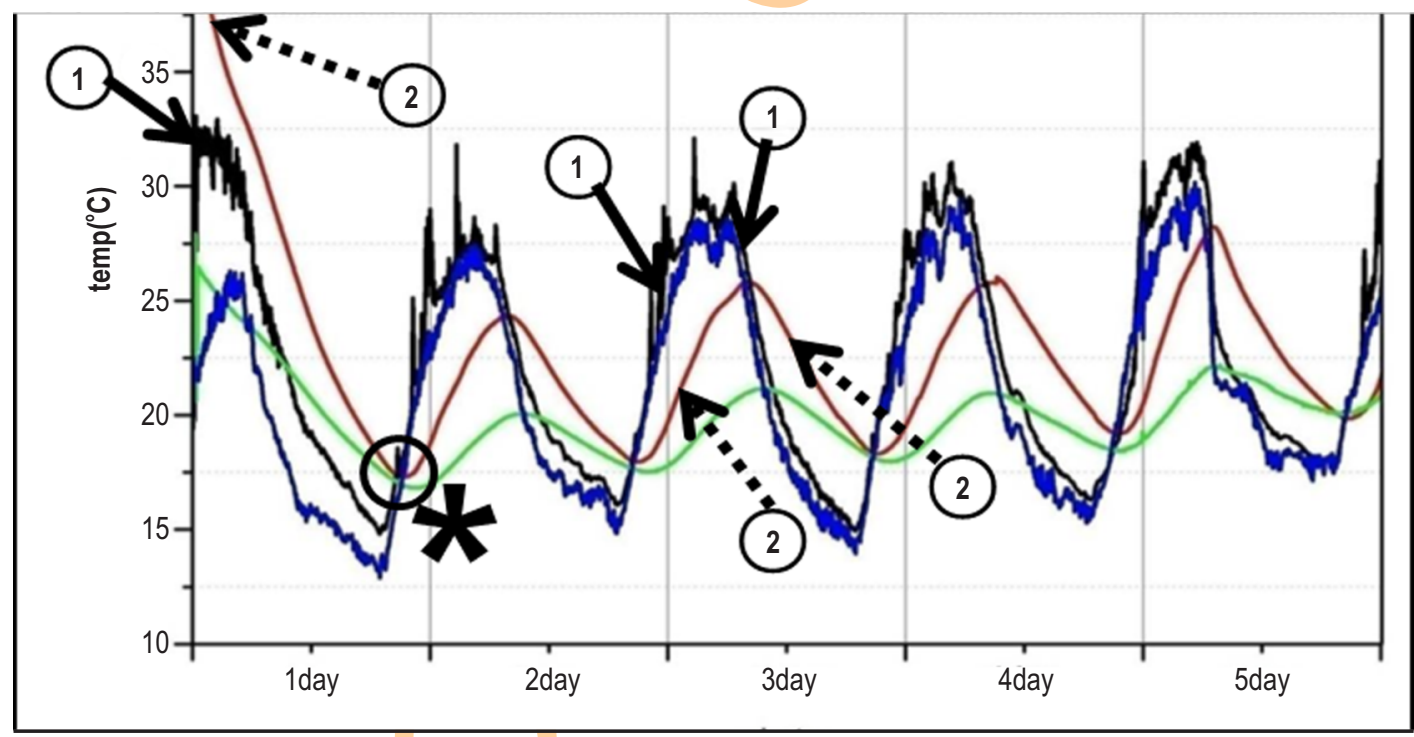

Fig . 3 : Results of cooling curve analysis for ambient temperature and rectal temperature. Rectal temperature (dashed arrow with (2)) began to fall when the experiment began. Once the rectal temperature reached (circle with *) that of the ambient temperature (arrow with (1)), the rectal temperature fluctuated thereafter. As the ambient temperature rose, the rectal temperature rose 2 to 3 hours after, and when the ambient temperature fell, the rectal temperature fell 2 to 4 hours after.

reached the ambient temperature, it used to be hard to increase the temperature of dummy. So there was no mention after the temperature reached the ambient temperature in the previous study. Also, this study showed that pigs can be good animal models that can substitute human cadaver to study the process of decomposition. Lastly, the rate of cooling of the body is represented by a cubic function of time rather than an exponential or bi-exponential function (Henssge, 1988). It would be helpful to create a novel formula for estimating time since death during the early stages of decomposition. 


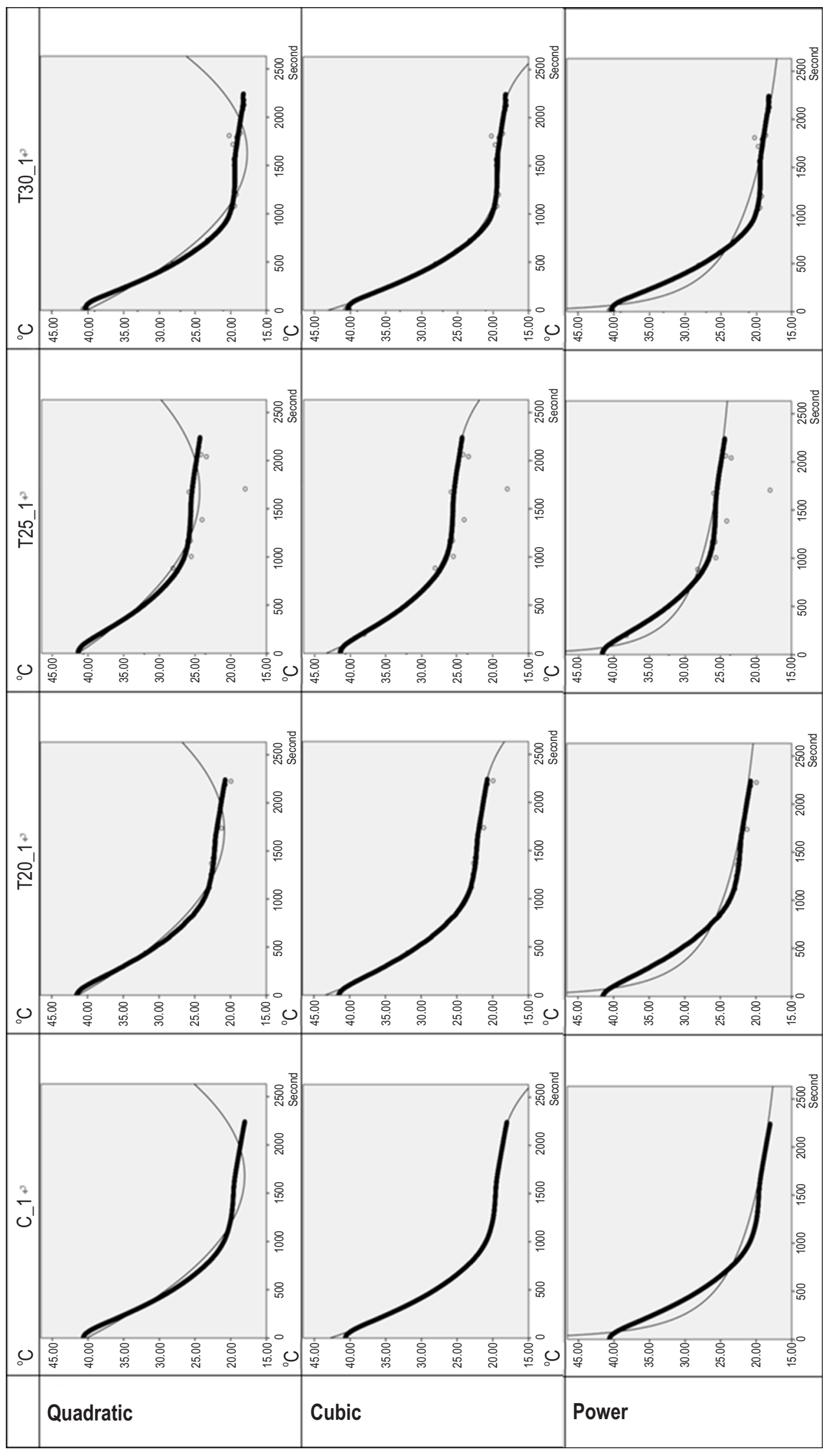

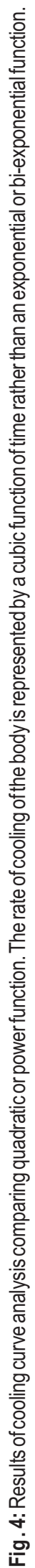




\section{Acknowledgment} University.

This work was supported by the Soonchunhyang

\section{References}

Althus, L. and C.Henssge: Rectal temperature time of death nomogram: Sudden change of ambient temperature. Anat. Anz., 99,171-178 (1999).

Henssge, C.: Rectal temperature time of death nomogram: Dependence of corrective factors on the body weight under stronger thermic insulation conditions. Forensic Sci. Int., 54, 51-66 (1992).

Henssge, C.: Death time estimation in case work. I. The rectal temperature time of death nomogram. Forensic Sci. Int., 38, 209-236 (1988).

Henssge, C., L. Althaus, J. Bolt, A. Freislederer, H.T. Haffner, C.A Henssge, B. Hoppe and V. Schneider: Experiences with a compound methodfor estimating the time since death. I. Rectal temperature nomogram for time since death. Int. J. Legal Med.,113, 303-319 (2000a).

Henssge, C., L. Althaus, J. Bolt, A. Freislederer, H.T. Haffner, C.A. Henssge, B. Hoppe and V. Schneider: Experiences with a compound methodfor estimating the time since death.II. Integration of non-temperature-based methods. Int. J. Legal Med., 113, 320-331 (2000b).

Henssge, $\mathrm{C}$. and B. Madea: Estimation of the time since death in the earlypost-mortem period. Forensic Sci. Int., 144, 167-175 (2004).

Henssge, C. and B. Madea: Estimation of the time since death. Forensic Sci. Int., 165, 182-184 (2007).

Marshall, T. and F. Hoare: Estimating the time of death. The rectal cooling after death and its mathematical expression. J. Forensic Sci. 7, 5691 (1962)

Megyesi, M., S. Nawrocki and N. Haskel: Using accumulated degreedays to estimate the postmortem interval from decomposed human remains. J. Forensic Sci., 50, 618-626 (2005). 